\title{
Proposal for the Implementation of the ALOHA Up-Conversion Interferometer on the CHARA Telescope Array
}

\author{
J. T. Gomes*, ${ }^{*}$, L. Grossard*, R. Baudoin*, L. Delage*, F. Reynaud*, \\ T. A. ten Brummelaar ${ }^{\dagger}$, N. J. Scott ${ }^{\dagger}$, J. Sturmann $^{\dagger}$ and V. Coudé du Foresto ${ }^{\ddagger}, \uparrow^{\natural}$ \\ *XLIM, Département Photonique \\ Université de Limoges, UMR CNRS 7252, \\ 123 Av. Albert Thomas, 87060 Limoges Cedex, France \\ ${ }^{\dagger}$ The CHARA array, Mount Wilson Observatory \\ Mount Wilson, CA 91023, USA \\ ${ }^{\ddagger}$ LESIA - CNRS, Observatoire de Paris \\ 92192 Meudon Cedex, France \\ §jean-thomas.gomes@unilim.fr
}

Received 2013 June 30; Revised 2014 September 12; Accepted 2014 October 15; Published 2014 November 28

\begin{abstract}
In this paper, we present a new concept of instrument for high resolution imaging in astronomy, involving the sum frequency generation in non-linear waveguides. The aim is to convert the infrared radiation emitted by an astronomical source to the visible spectral domain where the optical components are mature and efficient. We present the main experimental results obtained in laboratory, and propose a new design for this instrument for its implementation on the Center for High Angular Resolution Astronomy (CHARA) telescope array. Preliminary stability and photometric results obtained at CHARA are presented. Using these last measurements, we estimate the limiting magnitudes which could be reached by this interferometer in the $\mathrm{H}$ spectral band.
\end{abstract}

Keywords: Aperture synthesis, interferometry, nonlinear wave mixing, up-conversion, fiber optics.

\section{Introduction}

For more than 25 years, we are developing a new generation of instrument dedicated to high resolution imaging in astronomy. In this framework, we have contributed to the development of the use of optical fiber to link a telescope array to a central beam combining station while preserving the mutual coherence measurements. For a long time, optical fibers have been mainly developed for telecommunication applications in the $1.55 \mu \mathrm{m}$ window. This spectral region addresses the $\mathrm{J}$ and $\mathrm{H}$ astronomical bands and several projects have used silica fibers to develop new instrumental configurations (AMBER (Robbe-Dubois et al., 2007),

\footnotetext{
$\S_{\text {Corresponding author. }}$

ฯ Currently visiting scientist at the Center for Space and Habitability, Bern University.
}

OHANA (Woillez et al., 2001), MIRC (Monnier et al., 2006), etc.) dedicated to these spectral windows. To extend this spectral domain, instruments such as FLUOR (Coudé du Foresto \& Ridgway, 1992) uses more exotic fibers such as Fluoride fibers to address the $\mathrm{K}$ band. Unfortunately, no efficient fibers are currently available to extend this method to longer wavelengths in order to reach, for instance, the $\mathrm{L}$ and $\mathrm{M}$ bands.

For this reason, we proposed a completely different strategy in the framework of the Astronomical Light Optical Hybrid Analysis (ALOHA) project. Using a non-linear optical process, it could be possible to shift the wavelength of the astronomical light from the mid (MIR) or far (FIR) infrared to shorter wavelengths to take advantage of the silica fiber performance. This spectral shift can be achieved using sum frequency generation (SFG) process in non-linear waveguides. In this 
configuration, the frequency change has to take place at the focus of each telescope of the array. This way, the converted light reaches the silica fiber transparency window and allows the use of high performance guided optic components for beam transport, recombination and other optical functions such as spatial filtering.

For more than 10 years, we intend to validate this proposal through a set of laboratory experiments at XLIM. To minimize the experimental difficulties, these first experiments are conducted from the near infrared (NIR) to the visible spectral domains. Thanks to the current experimental results, we are now ready to plan a real experiment on sky in the frame of a collaboration with the Center for High Angular Resolution Astronomy (CHARA) telescope array (Brummelaar et al., 2005). The aim of this paper is to describe the proposal about the implementation of the ALOHA up-conversion interferometer on the CHARA array.

In the first part of this paper, we report on the principle and the preliminary experimental results obtained with our up-conversion interferometer. In order to validate this concept, the second part shows how we plan to implement the instrument on the CHARA array. Preliminary results are reported in order to evaluate the vibration environment and the coupling efficiency from the telescope to our experimental setup input. Using the previously obtained results, the last part summarizes the potential of the ALOHA/CHARA setup through the estimation of the limiting magnitude which could be reached in $\mathrm{H}$ band.

\section{The ALOHA Concept}

Figure 1 shows the main proposals used in the context of aperture synthesis for optical astronomy in order to give a good overview of the framework of the ALOHA concept.

Over all these techniques, the main feature is to get a cross correlation of the light waves received by the different telescopes of the array. This way, the object is analyzed through the sampling of its spatial spectrum at different spatial frequencies. Depending on the method, these correlations processes are applied either on the optical fields or on the intensity.

In the Fizeau Michelson Labeyrie configuration (Fig. 1(a)), the interference allows to get the cross correlation between the optical fields. The contrast measurement and the phase closure acquisition give access to the complex visibility. The experimental results can be directly used for model fitting. When an image reconstruction is preferred, reconstruction algorithms are used to process the data. This configuration is the most common technique currently applied on many instruments such as the VLTI, NPOI, CHARA, etc.

In 1956, Brown and Twiss proposed to make the coherence analysis using the correlation of the intensity detector at the focus of two telescopes (Fig. 1(b)). The cross correlation of the detector output electric signal provides the information on the mutual coherence of the optical intensity related to the object spatial spectrum. Nevertheless the limited narrow bandwidth of the electric signal processing strongly limits the sensitivity of this instrument.

To overcome this limitation, Townes developed the heterodyne interferometry by mixing the astronomical lights with a powerful laser to get a higher sensitivity (Fig. 1(c)). However, if the heterodyne configuration is able to enhance the performance of this instrument, the electric correlator remains the limiting point. The electronics are not able to process the very large bandwidth of astronomical optical fields.

To avoid this main limitation, we proposed in 2008 a new instrumental concept (Del Rio et al., 2008). This setup, shown in Fig. 1(d), takes advantage from 1 (a) and 1(c) configurations:

- by using a local oscillator to mix the astronomical light with a powerful laser operating in the optical domain, it is possible to preserve a large spectral operation bandwidth,

- by making a cross correlation of the optical fields rather than the optical intensity, this instrument allows to preserve the large bandwidth of the optical fields correlation.

In our experimental configuration, we use SFG process. The pump laser at $1064 \mathrm{~nm}$ (DFB laser) shifts the infrared input optical spectrum (around $1550 \mathrm{~nm})$ to a visible optical radiation $(633 \mathrm{~nm})$, conversely to the Townes' configuration where the wave mixing provides a signal in the low frequency electrical domain. This fully optical light-to-light processing allows to operate with a spectral bandwidth much larger than in the intensity correlation configurations 1 (b) and 1(c).

This wavelength shift is obtained through a non-linear crystal (Boyd, 2003), where each 


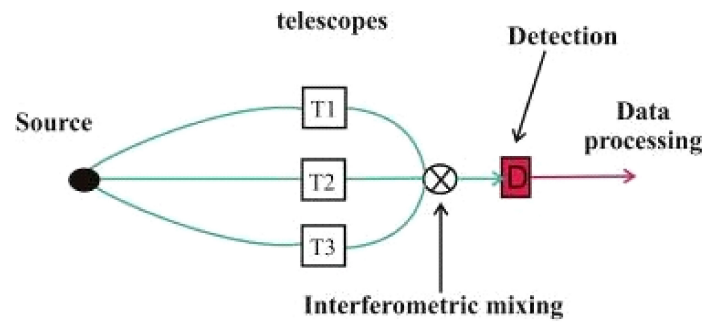

- Optical signals

- Electronic signals

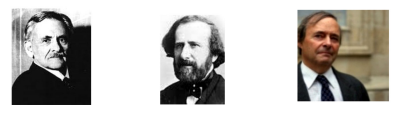

(a) Michelson Fizeau Labeyrie

Local oscillator
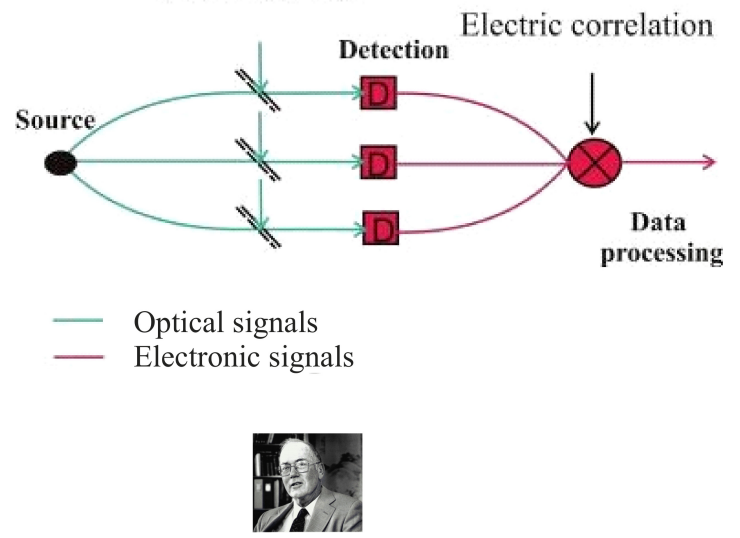

(c) Townes

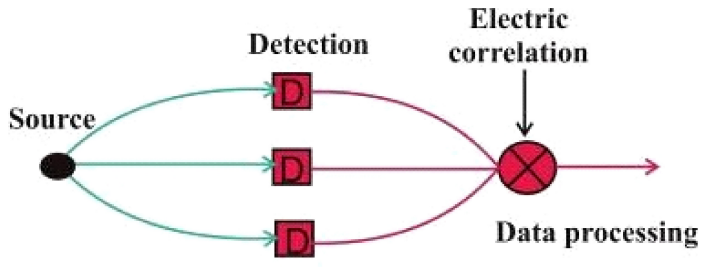

- Optical signals
Electronic signal



(b) Brown \& Twiss

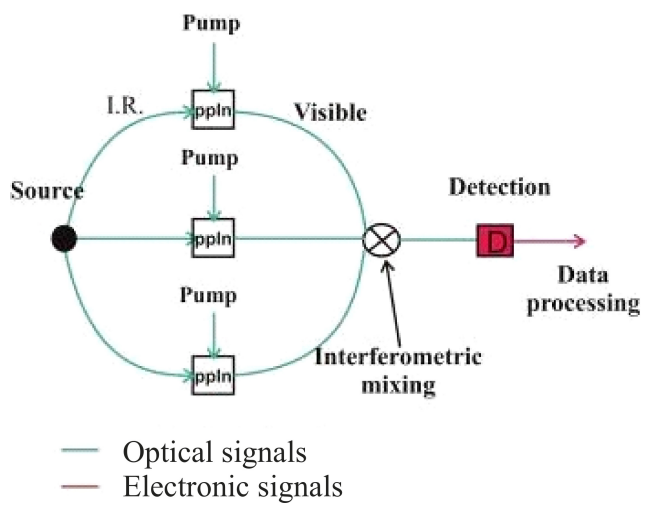

(d) XLIM

Fig. 1. Main proposals for aperture synthesis applied to astronomy.

converted photon in the visible range results from the superimposition of a pump photon with a scientific signal one. This non-linear coherent process allows to transfer the phase information from the scientific infrared signal to the converted wave. The SFG, well known to be intrinsically noiseless (Louisell et al., 1961), is fully compliant with our stringent high dynamic experimental configuration, where the powerful pump laser $(30 \mathrm{~mW})$ is mixed with the very faint astronomical source (few fW). In this framework, we have recently demonstrated, in lab, that it is possible to convert the faint radiation emitted by a blackbody source (acting as an astrophysical source) using a powerful laser pump, despite the huge intensity mismatch between the two waves to be mixed (Gomes et al., 2014).

Doing so, it is possible to shift the original infrared light to a spectral domain more convenient for the instrument implementation. A very good trade-off is to convert the light within the 600 to $900 \mathrm{~nm}$ spectral band. This way it is possible to propose an experimental design using very efficient components.

In this spectral domain, Silicon photon counting detectors are well known to be very efficient devices with a very high sensitivity $(70 \%)$ and a very low dark count level ( 20 counts/s). Moreover, the use of singlemode waveguides allows a very good calibration of the fringe visibility. This property has been intensively used on instruments such as FLUOR, AMBER and MIRC (Coudé du Foresto \& Ridgway, 1992; Robbe-Dubois et al., 2007; Monnier et al., 2006). In addition, optical fibers are more efficient transmission media with the lowest propagation losses. The OHANA project (Woillez et al., 2001) takes advantage of this property to propose a very long base interferometer on the Mauna Kea. 


\section{Laboratory Experimental and Sensitivity Preliminary Results}

Although the principle of ALOHA is very attractive, the involved non-linear processes may induce disturbances on the optical fields, leading to a modification of the mutual coherence of the beams collected by the telescopes of the array. Therefore, an experimental study is to be carried out to quantify the reliability of the measurements using the up-conversion interferometer. Performing this investigation, it is possible to take advantage of many efficient optical components previously developed mainly for optical telecommunications. For this reason, all the experimental results reported below have been obtained in the $1.55 \mu \mathrm{m}$ spectral domain. Future developments are planned to reach the MIR and FIR spectral domains to enhance the scope of potential applications.

Figure 2 shows the generic structure of the instrument used for all our laboratory investigations. The first main result is reported in Brustlein et al. (2008). For this experiment, we implemented a laboratory prototype of a telescope array simultaneously connected to a classical infrared and an up-conversion interferometer, including a SFG process on each interferometric arm. This experimental setup allowed to observe simultaneously the fringe contrasts with these two instruments. Using a laboratory binary star as a source and changing the baseline between the two input telescopes of the array, it has been possible to demonstrate the very good agreement between the contrast measurements obtained from the classical interferometer and those obtained with our new instrument. These results have been obtained with a laser source and the flux level was far higher than the astronomical requirements.

The second observable to be acquired is the phase closure. For this purpose, we modified the previous interferometer to add a third interferometric arm. The calibrated Multi Aperture Fiber Link (MALF) interferometer was used as the IR reference instrument. This prototype, developed for ESA in collaboration with IMEP, GEEO and Matra Marconi Space, uses an integrated component and is very reliable $(0.1 \%$ contrast accuracy and 0.01 rad phase closure accuracy) (Olivier et al., 2007). Using a fixed telescope configuration and changing the power ratio between the two components of a laboratory binary star, it has been possible to scan a large domain of phase closure values. Ceus et al. (2011) reports on the very good accuracy of the SFG interferometer measurements using the MAFL instrument as a reference. Nevertheless, these last measurements were always performed at high flux level.

The third point experimentally studied was the possibility to operate a SFG interferometer at very low flux down to the photon-counting regime. This experiment used the previous threearm interferometer with the possibility to significantly attenuate the optical fluxes routed to the SFG interferometer. The MAFL always operated at high flux level to preserve a very high accuracy of the reference contrasts and phase closure measurements, while our instrument under test worked in the photon counting regime. The data processing involved the Wirnitzer correction (Longueteau et al., 2003) in order to correct the photon noise biases on the two observables. The related

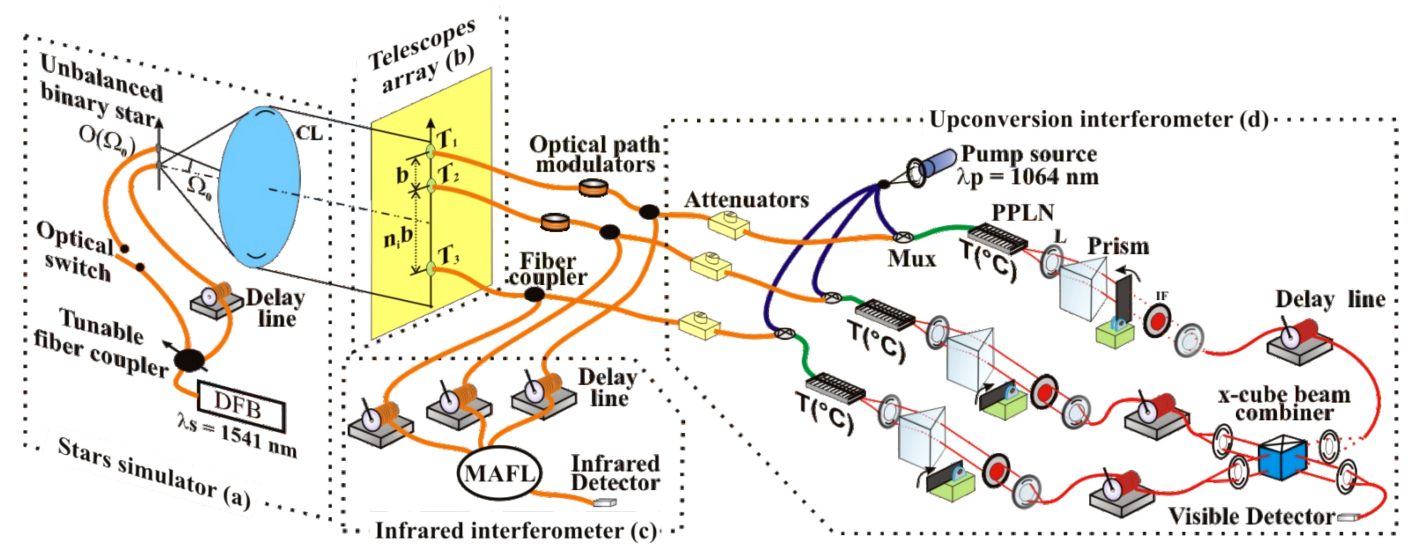

Fig. 2. Generic scheme of our three-arm up-conversion interferometer working with an imaging bench. This instrument works in parallel with a fibered infrared interferometer providing reference results. 
experimental results demonstrated the possibility to make accurate measurements down to 200 photons per measurement frame (Ceus et al., 2013). This raw value can be easily improved mainly by a convenient data processing as recently demonstrated in Gomes et al. (2014).

The last major result is reported in Ceus et al. (2012). To scale-up our experimental setup to the astronomical observing conditions, we performed an experimental demonstration on the sky with a single interferometric arm. The light was collected by a $203 \mathrm{~mm}$ diameter telescope (Celestron C8) and then was converted through a SFG process from the $\mathrm{H}$ band into the visible domain. Despite the very small size of the telescope aperture and the very low coupling efficiency, it was possible to detect the light of Arcturus, Betelgeuse and Pollux in photon counting regime.

Thanks to these experimental demonstrations, we are now able to propose a visiting instrument compliant with the CHARA telescope array.

\section{Implementation on CHARA}

\subsection{Coupling assembly interface with FLUOR}

The implementation of the ALOHA instrument on the CHARA array uses the Fiber Linked Unit of Optical Recombination (FLUOR) instrument (Coudé du Foresto et al., 2003). This instrument is a two-arm fiber-based beam combiner. It specializes in high precision measurements of interferometric visibility. Any pair of the six CHARA telescopes can be directed to the FLUOR optical bench giving access to baselines from 34 to $331 \mathrm{~m}$. The FLUOR optical path provides static and dynamic optical path delays. FLUOR also provides alignment optics and cameras for viewing the beam image and pupil plane in $\mathrm{V}$ and $\mathrm{H}$ bands, respectively. Upon reaching the FLUOR bench the beam passes a pair of dihedral mirrors and one fold mirror before falling upon a tip-tilt stage. This stage directs light onto a 1.5 inch diameter $\mathrm{f} / 1.330^{\circ}$ off-axis parabola (OAP). The tip-tilt mounts have a repeatable position accuracy of 1.8 arc-seconds which represent approximately two micrometers on the fiber head plan.

At the focus of the OAP is a XYZ translation stage holding a E2000 fiber connector. The E2000 connectors are known for providing micronlevel precision for fiber placement. For ALOHA operation, the FLUOR optical fibers will be disconnected and replaced by the ALOHA fiber feeds. This parallel setup enables easy switching between the two instruments and gives ALOHA the benefits of FLUOR's alignment and integration with the CHARA array. FLUOR's software controlled OPD and tip-tilt stages can be used to provide additional optical delay and to perform raster scans to optimize the injection of light into the fibers.

\subsection{Proposed design for the ALOHA instrument}

The ALOHA instrument is shown in Fig. 3. On this setup, the beams incoming from the FLUOR coupling assembly and a laser pump source are spectrally multiplexed thanks to polarization maintaining fibered wavelength division multiplexers (WDM). This pump source provides the energy required to reach the SFG process in each interferometric arm. The emerging multiplexed beams pass through achromatic injection systems to feed the non-linear crystals.

The non-linear processes take place in $40 \mathrm{~mm}$ long specially designed Ti:PPLN (Periodically Poled Lithium Niobate) waveguides with a spectral acceptance of $0.3 \mathrm{~nm}$. These crystals are characterized by a poling periodicity $\Lambda=10.85 \mu \mathrm{m}$. Using a pump source at $1064 \mathrm{~nm}$, they allow to convert a $1550 \mathrm{~nm}$ radiation to $630 \mathrm{~nm}$ at an operating temperature equal to $90^{\circ} \mathrm{C}$. This temperature is controlled on each PPLN waveguide by thermally regulated enclosures. The thermal stability is in the range of $0.01^{\circ} \mathrm{C}$, which permits a very good overlapping of the spectral acceptances of the PPLN in the two arms of the interferometer. This leads to a symmetric quasi-phase matching leading to the same SFG process on each interferometric arm.

The emerging up-converted signals at visible wavelengths are spectrally selected on each arm by means of a spectral filtering stage, made up of a dispersive prism $(\mathrm{P})$ and an interference filter (IF) centered on the mean converted wavelength. After this stage, the converted signals are spatially filtered thanks to singlemode polarization maintaining fibers at the converted wavelength. A $10-\mathrm{cm}$ stroke fibered delay line is inserted in the arm 2 to adjust the optical path difference (OPD) between the two arms of the interferometer (Simohamed et al., 1996). An optical path modulator (OPM) (Reynaud \& Delaire, 1993), with a 


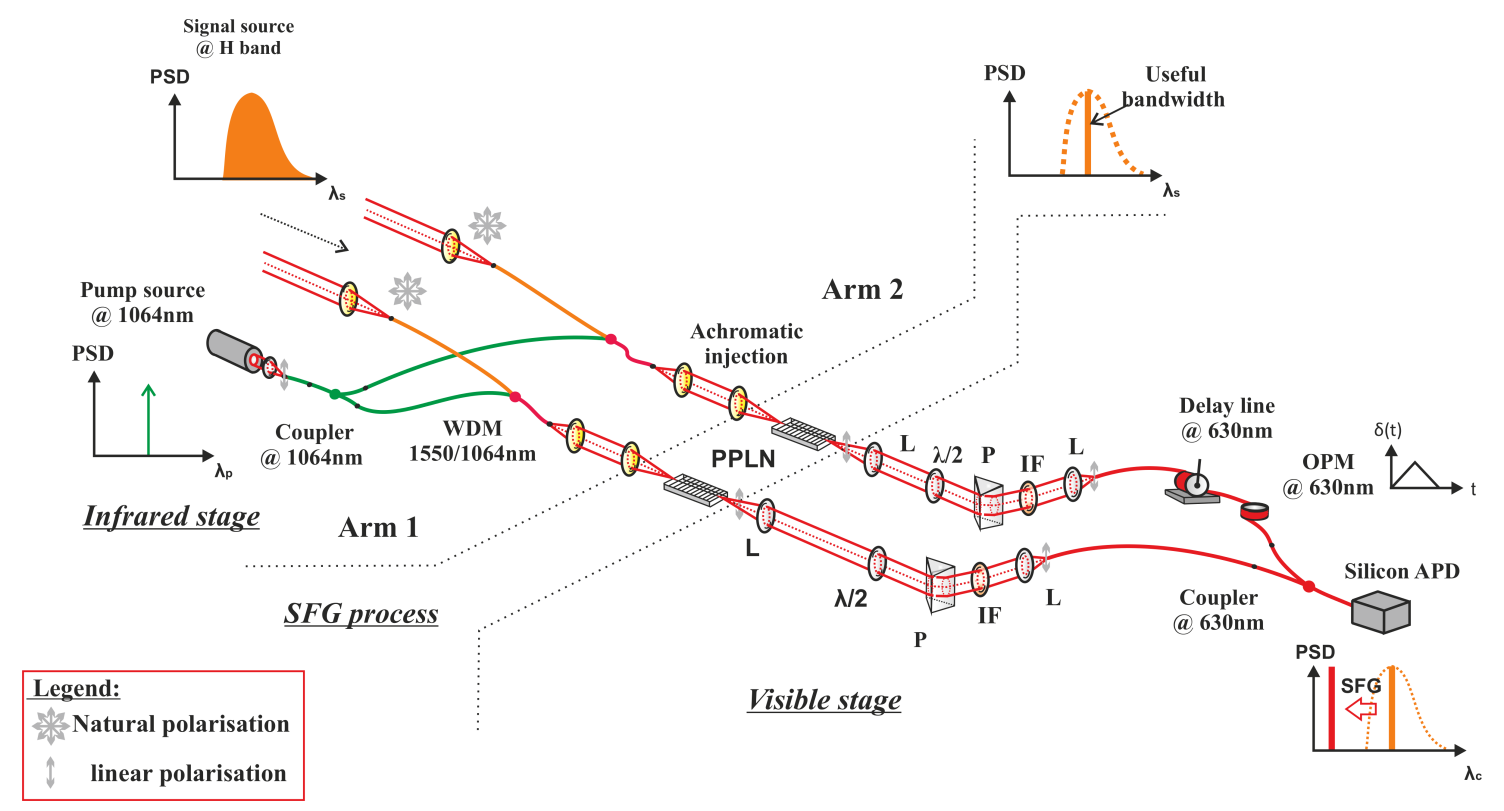

Fig. 3. Global scheme of the ALOHA instrument which can be connected to the FLUOR coupling assembly interface. PSD: power spectral density, WDM: wavelength division multiplexer, L: lens, P: prism, IF: interference filter, OPM: optical path modulation.

$100 \mu \mathrm{m}$ stroke, is inserted in the same arm for a fine OPD adjustment and to induce a temporal optical path modulation on the converted signal. This allows to display the fringe pattern as a function of time. This device is driven by a triangular high voltage to induce temporal sequenced linear OPDs. At the output of the up-conversion interferometer, we combine the two visible optical fields through a polarization maintaining and singlemode fiber coupler. The resulting fringe pattern is then detected by a Silicon avalanche photodiode (Si-APD) operating in the photon counting regime.

\subsection{Environmental tests and sensitivity evaluation}

To plan the implementation of ALOHA at the CHARA array, we achieved a preliminary mission on the array site. This mission had two main goals:

- to check the vibration and thermal environments on site and their possible impact on the ALOHA instrument,

- to achieve photometric measurements at the output of the FLUOR coupling assembly interface in order to have a better knowledge of the CHARA array throughput and the available light level at the input of ALOHA fibers.
For the calibration of vibration and thermal environments at the CHARA array site, we have implemented a reference fibered Mach Zehnder interferometer working in $\mathrm{H}$ band. Through the comparison of interference fringes obtained with a laser diode $\left(\lambda_{s}=1550 \mathrm{~nm}, \Delta \lambda_{s}=80 \mathrm{~nm}\right)$ in laboratory and on the CHARA site, we can determine the environmental condition for ALOHA instrument implemented at CHARA.

Figure 4 shows the fringe pattern obtained with our reference interferometer at the XLIM laboratory in Limoges on 2013 March 14 (left curve) and the same one observed at CHARA observation site on 2013 March 23 (right curve). These matching results show that the environmental conditions at CHARA are the same than in our laboratory. This way, the result that we will obtain on the ALOHA instrument during the laboratory test phase will be transferable on the telescope array site without any adjustment.

A throughput estimation from a telescope of the CHARA array to the output of the FLUOR coupling assembly interface was achieved during the night of 2013 March 26. During this measurement, the light collected by the telescope observing Arcturus (magnitude -2.81 in $\mathrm{H}$ band) was injected successively in a multimode fiber and in a singlemode polarization maintaining fiber at $1550 \mathrm{~nm}$ placed at the output of the coupling assembly 

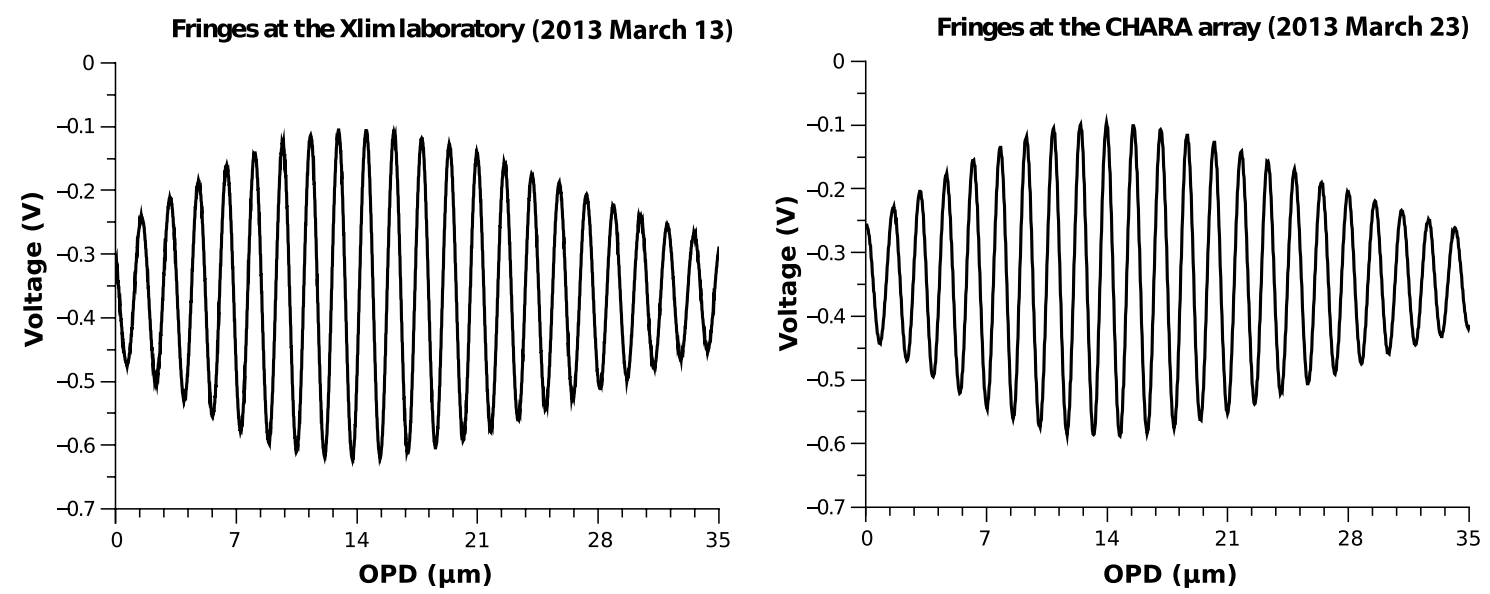

Fig. 4. Left: interference fringes observed in lab on 2013 March 13. Right: interference fringes observed at CHARA on 2013 March 23.



Fig. 5. (Color online) Blue curve: intensity detected at the output of a multimode fiber connected to the FLUOR launching assembly interface. We measured a mean flux at the fiber output $P_{\text {multi }}=61.1 \mathrm{pW}$. Red curve: the same one with a singlemode polarization maintaining fiber at $1550 \mathrm{~nm}$. We measured a mean flux at the fiber output $P_{\text {mono }}=7.1 \mathrm{pW}$ (note that the detector noise is $1.8 \mathrm{pW}$ ).

interface. Through the detection of the light at the fiber output, we can estimate the fiber coupling efficiency at the input of ALOHA instrument.

Figure 5 shows the photometric levels that we have observed by pointing Arcturus during 2013 March 25 night. With the astrophysics signal off, the power level is equal to the $1.8 \mathrm{pW}$ detector noise (not shown in the figure). We have measured a mean flux $P_{\text {multi }}=61.1 \mathrm{pW}$ at the multimode fiber output and $P_{\text {mono }}=7.1 \mathrm{pW}$ at the singlemode fiber output. Each measurement point results from an integration over $1 \mathrm{~s}$. These results are used in the next part to derive a prospective evaluation on the ALOHA potential performances operating on the CHARA site.

\section{Prospective and Discussion}

We propose below a prospective evaluation of the sensitivity of ALOHA applied on the CHARA array.

\subsection{Evaluation of the overall transmission}

\subsubsection{Current performance}

The global throughput $T$ of ALOHA implemented on the CHARA array is a combination of three main contributions (see Fig. 6):

$T_{1}$ : the intensity throughput from the telescope to the ALOHA instrument,

$T_{2}$ : the fiber coupling efficiency,

$T_{3}$ : the global transmission of the ALOHA instrument.

$T_{1}$ is evaluated from the transmission coefficient of each component included in the optical linkage between the telescope and the output of the delay lines. According to the CHARA team, $T_{1}$ is estimated to $40 \%$ of the input light reaching the telescopes. This value only takes the intensity throughput into account but does not include the wavefront distortions that will be cleared through the spatial filtering $\left(T_{2}\right)$.

$T_{2}$ results from the overlapping of the wavefront outcoming from the delay lines with the singlemode field to be propagated in the optical fibers operating in the $\mathrm{H}$ band. $T_{2}$ is evaluated in Fig. 5 on a real object by measuring the ratio between the flux collected with a singlemode fiber (spatial filtering) and the one injected in a multimode fiber (no spatial filtering, assuming the seeing smaller than the core diameter). Observations on Arcturus $\left(-2.81 \mathrm{H}_{\mathrm{mag}}\right)$ 


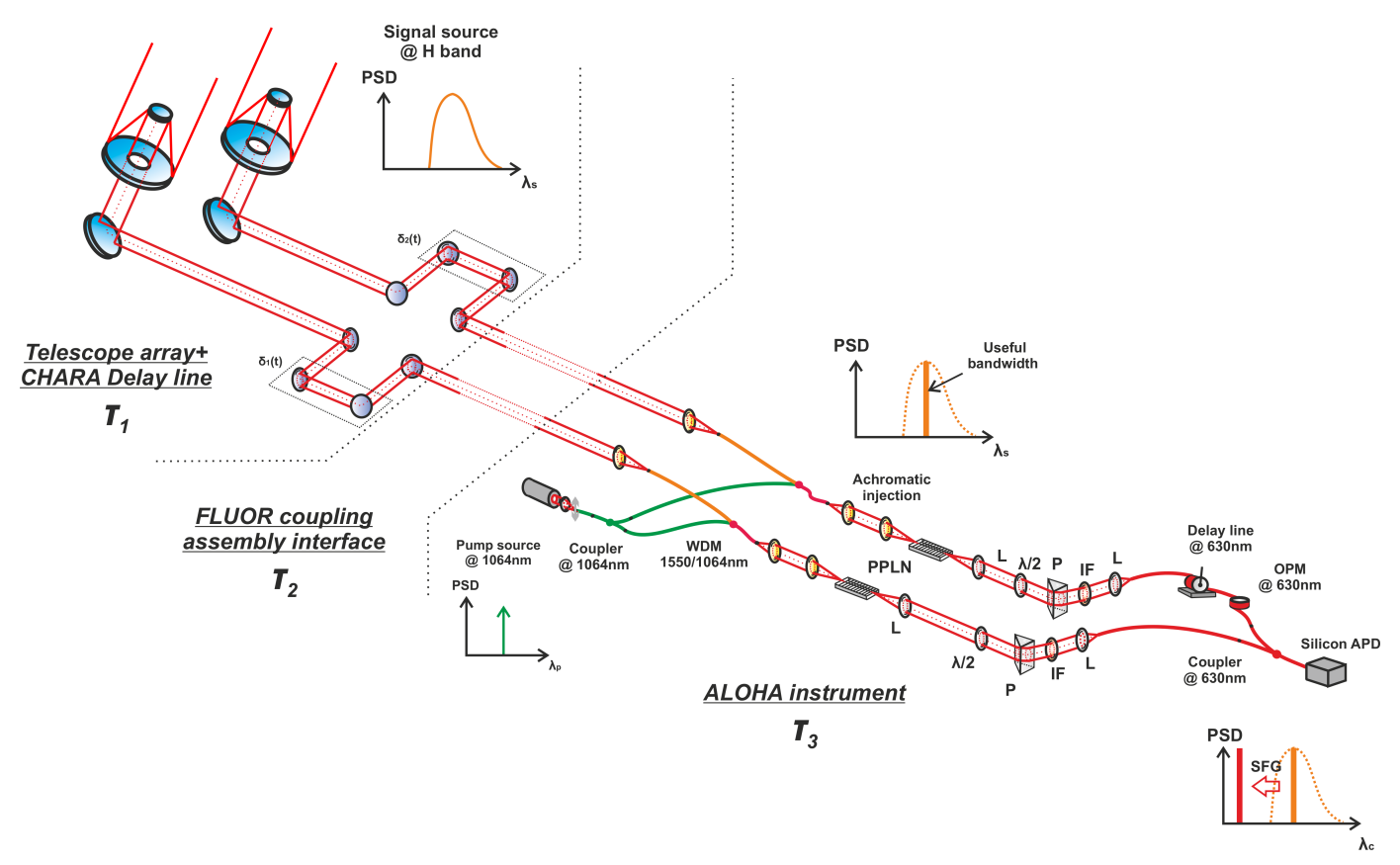

Fig. 6. Transmission coefficients of the CHARA/ALOHA instrument.

with a seeing in the range of $10-20 \mathrm{~cm}$ allow to obtain $T_{2}=11.6 \%$. This estimation takes the telescope central obstruction into account.

$T_{3}$ results from the contributions of:

- the multiplexer transmission coefficient,

- the SFG module global efficiency,

- the visible optical mixer throughput,

- the detection efficiency.

The measured global transmission has been characterized in laboratory and is equal to $T_{3}=$ $0.2 \%$. These results allow to compute the global transmission coefficient of ALOHA implemented on the CHARA array: $T=T_{1} \cdot T_{2} \cdot T_{3}=9.3 \cdot 10^{-3} \%$. Notice that this global transmission coefficient is related to the spectral analysis performed in the SFG stage with a $R=5000$ resolving power.

\subsubsection{Expected upgraded performance}

$T_{1}$ is constant as long as the CHARA array is currently operating for astrophysics measurements and minor changes are foreseen. $T_{2}$ will be improved with the implementation of adaptive optics. According to Coudé du Foresto et al. (2000), the throughput could be enhanced significantly to get $T_{2}$ close to $60 \% . T_{3}$ can be significantly upgraded. The main issue is the conversion efficiency of the SFG module. Two ways can be followed:
- using a frequency comb as a pump source for the SFG process. In this configuration, each line of the pump source addresses a different part of the spectrum of the source under analysis, increasing the spectral bandwidth which can be used by the ALOHA instrument. Considering that each line of the frequency comb presents the same intensity, the conversion efficiency improvement is then proportional to the number of lines in the frequency comb but reduces the resolving power,

- using a pigtailed device to improve input and output coupling between the singlemode fiber and the non-linear crystal. Using the data reported in Shentu et al. (2013), it is reasonable to reach a $T_{3}$ coefficient equal to $3.1 \%$.

According to this assumption, it is possible to derive the global transmission as reported in Table 1.

\subsection{Evaluation of the limiting magnitude vs. experimental assumptions}

The limiting magnitude of ALOHA implemented on the CHARA array is driven by the global throughput, the surface of the telescope and the noise of the detector (dark-counts per second). Commercial detectors can easily reach a dark count level equal to 20 photons per second. This value will 
Table 1. Global transmission of CHARA/ALOHA as a function of the instrument configuration.

\begin{tabular}{|c|c|c|c|c|c|c|}
\hline \multicolumn{2}{|c|}{$\begin{array}{l}\text { Instrument } \\
\text { configuration }\end{array}$} & \multirow{2}{*}{$\begin{array}{c}\text { Spectral } \\
\text { bandwidth }(\mathrm{nm})\end{array}$} & \multicolumn{4}{|c|}{$\begin{array}{c}\text { CHARA/ALOHA } \\
\text { transmission }(\%)\end{array}$} \\
\hline CHARA & ALOHA & & $T_{1}$ & $T_{2}$ & $T_{3}$ & $T$ \\
\hline \multirow[t]{3}{*}{ No adaptive optics } & $\begin{array}{l}\text { Current } \\
\quad(1 \text { pump line })\end{array}$ & 0.30 & 40.0 & 11.6 & 0.2 & $9.3 \cdot 10^{-3}$ \\
\hline & $\begin{array}{l}\text { Current } \\
\quad(4 \text { pump lines })\end{array}$ & 1.20 & 40.0 & 11.6 & 0.2 & $9.3 \cdot 10^{-3}$ \\
\hline & $\begin{array}{l}\text { Fibered PPLN } \\
\quad(1 \text { pump line })\end{array}$ & 0.16 & 40.0 & 11.6 & 3.1 & 0.14 \\
\hline \multirow[t]{3}{*}{ Adaptive optics } & $\begin{array}{l}\text { Current } \\
\quad(1 \text { pump line })\end{array}$ & 0.30 & 40.0 & 60.0 & 0.2 & $4.8 \cdot 10^{-2}$ \\
\hline & $\begin{array}{l}\text { Current } \\
\quad \text { (4 pump lines })\end{array}$ & 1.20 & 40.0 & 60.0 & 0.2 & $4.8 \cdot 10^{-2}$ \\
\hline & $\begin{array}{l}\text { Fibered PPLN } \\
\text { (1 pump line })\end{array}$ & 0.16 & 40.0 & 60.0 & 3.1 & 0.74 \\
\hline
\end{tabular}

Table 2. Limiting magnitude of CHARA/ALOHA as a function of the instrument configuration.

\begin{tabular}{|c|c|c|c|}
\hline \multicolumn{2}{|c|}{$\begin{array}{l}\text { Instrument } \\
\text { configuration }\end{array}$} & \multirow{2}{*}{$\begin{array}{c}\text { Spectral } \\
\text { bandwidth }(\mathrm{nm})\end{array}$} & \multirow{2}{*}{$\begin{array}{l}\text { Limiting magnitude } \\
\qquad(\mathrm{H} \text { band })\end{array}$} \\
\hline CHARA & ALOHA & & \\
\hline \multirow[t]{3}{*}{ No adaptive optics } & $\begin{array}{l}\text { Current } \\
\text { (1 pump line) }\end{array}$ & 0.30 & 1.6 \\
\hline & $\begin{array}{l}\text { Current } \\
\text { (4 pump lines) }\end{array}$ & 1.20 & 3.2 \\
\hline & $\begin{array}{l}\text { Fibered PPLN } \\
\quad(1 \text { pump line })\end{array}$ & 0.16 & 3.5 \\
\hline \multirow[t]{3}{*}{ Adaptive optics } & $\begin{array}{l}\text { Current } \\
\text { (1 pump line })\end{array}$ & 0.30 & 3.4 \\
\hline & $\begin{array}{l}\text { Current } \\
\quad \text { (4 pump lines) }\end{array}$ & 1.20 & 4.9 \\
\hline & $\begin{array}{l}\text { Fibered PPLN } \\
\quad(1 \text { pump line })\end{array}$ & 0.16 & 5.3 \\
\hline
\end{tabular}

be used in the following of this paper. Using the different configurations proposed in Table 1, it is possible to infer the corresponding limiting magnitude reported in Table 2 .

It is important to notice that these results do not take any signal processing into account to improve the signal to noise ratio (SNR). The reported magnitudes correspond to single snapshot measurements with a SNR equal to one. Moreover, in our experimental configuration, the coherence length of the converted light is directly related to the spectral acceptance of the nonlinear process. We experimentally obtain a value in the range of a few millimeters. This configuration allows to use averaging processes to improve the SNR and then enhance significantly the limiting magnitude.

It is noticeable that our instrument is not yet fully technologically mature. The aim of these tests is to show the feasibility of this new technique. The related performance has to be compared with the one of the CHARA instrument at the beginning of their implementation when the instruments were not optimized. 


\section{Conclusion}

In this paper, we have presented how the ALOHA instrument can be implemented on the CHARA array. This new kind of instrument uses SFG in PPLN waveguides to shift the signal wavelength from the IR domain to the visible one. This nonlinear effect is intrinsically noiseless and allows us to use mature and efficient optical components for transport and detection of light.

In previous laboratory studies, we demonstrated the potential of the up-conversion interferometer, in terms of fringe visibility and phase closure measurements. We also showed that the ALOHA interferometer can operate in the photon counting regime. Finally, we successfully converted the light from Arcturus, Betelgeuse and Pollux using one arm of the interferometer, demonstrating the scalability of the instrument to the astronomical observing conditions.

Through this paper, we propose a new design for the ALOHA instrument which can be connected to the existing FLUOR coupling assembly interface. A preliminary mission at CHARA allowed us to check the compatibility of the instrument with the vibration and thermal environments on site. We also achieved photometric measurements at the output of the FLUOR coupling assembly interface to evaluate the light levels we can expect at the input of ALOHA. From these preliminary results, we estimated the global transmission coefficient of ALOHA implemented on the CHARA array. Using fibered PPLN waveguides, the upgraded ALOHA/CHARA setup allows to reach a limiting magnitude up to 5.3 in the $\mathrm{H}$ band with a high resolving power $R=9375$. In the other hand, the use of a four line pump frequency comb to supply the non-linear processes increases the limiting magnitude up to 6.4 with a lower resolving power $R=1250$. These estimated values can be significantly enhanced by improving the SNR with an averaging process, making the ALOHA instrument a very competitive device in the frame of high resolution imaging in astronomy.

The improvement of the ALOHA performance is a key point in a next future in $\mathrm{H}$ band but we want to recall that the $\mathrm{H}$ band has been only selected for technological consideration. The main potential of ALOHA will be in the MIR and FIR where this technique will be more demonstrative.

\section{Acknowledgments}

This work has been financially supported by the Centre National d'Études Spatiales (CNES) and by the Institut National des Sciences de l'Univers (INSU). Our thanks go to A. Dexet for the development and his advices for all the specific mechanical components.

The CHARA array, operated by Georgia State University, was built with funding provided by the National Science Foundation, Georgia State University, the W. M. Keck Foundation, and the David and Lucile Packard Foundation. The CHARA array is currently funded by the National Science Foundation under Grant AST-0606958.

\section{References}

Boyd, R. W. [2003] Nonlinear Optics (Academic Press, London), p. 84.

Brummelaar, T. A. t., McAlister, H. A., Ridgway, S. T. et al. [2005] "First results from the CHARA array. II. A description of the instrument," ApJ. 628, 453.

Brustlein, S., Del Rio, L., Tonello, A. et al. [2008] "Laboratory demonstration of an infrared-to-visible upconversion interferometer for spatial coherence analysis," Phys. Rev. Lett. 100, 153903.

Ceus, D., Delage, L., Grossard, L. et al. [2013] "Contrast and phase closure acquisitions in photon counting regime using a frequency upconversion interferometer for high angular resolution imaging," MRNAS 430(3), 1529-1537.

Ceus, D., Reynaud, F., Woillez, J. et al. [2012] "Application of frequency conversion of starlight to high-resolution imaging interferometry. On-sky sensitivity test of a single arm of the interferometer," MNRAS 427, L95-L98.

Ceus, D., Tonello, A., Grossard, L. et al. [2011] "Phase closure retrieval in an infrared-to-visible upconversion interferometer for high resolution astronomical imaging," Opt. Exp. 19, 8616.

Coudé du Foresto, V., Borde, P. J., Merand, A. et al. [2003] "FLUOR fibered beam combiner at the CHARA array," in Proc. SPIE, Vol. 4838, p. 280.

Coudé du Foresto, V., Faucherre, M., Hubin, N. \& Gitton, P. [2000] "Using single-mode fibers to monitor fast Strehl ratio fluctuations," $A \& A \mathbf{1 4 5}, 305$.

Coudé du Foresto, V. \& Ridgway, S. T. [1992] "Fluor A stellar interferometer using single-mode fibers," in European Southern Observatory Conf. Workshop Proc., p. 731 .

Del Rio, L., Ribiere, M., Delage, L. \& Reynaud, F. [2008] "First demonstration of a temporal coherence analysis through a parametric interferometer," Opt. Commun. 281, 2722.

Gomes, J.-T., Delage, L., Baudoin, R. et al. [2014] "Laboratory demonstration of spatial coherence analysis of a blackbody through an up-conversion interferometer," 
Phys. Rev. Lett. 112, 143904, doi: 10.1103/PhysRevLett. 112.143904.

Longueteau, E., Delage, L. \& Reynaud, F. [2003] "Validation of photon noise correction using the OAST stellar interferometer breadboard," Opt. Commun. 217, 75.

Louisell, W. H., Yariv, A. \& Siegman, A. E. [1961] "Quantum fluctuations and noise in parametric processes. I." Phys. Rev. 124, 1646.

Monnier, J. D., Pedretti, E., Thureau, N. et al. [2006] "Michigan Infrared Combiner (MIRC): commissioning results at the CHARA Array," in Proc. SPIE, Vol. 6268, $62681 \mathrm{P}$.

Olivier, S., Delage, L., Reynaud, F. et al. [2007] "MAFL experiment: development of photonic devices for a spacebased multiaperture fiber-linked interferometer," Appl. Opt. 46, 834 .
Reynaud, F. \& Delaire, E. [1993] "Linear optical path modulation with $\lambda / 200$ accuracy using a fibre stretcher," Electron. Lett. 29, 1718.

Robbe-Dubois, S. et al. [2007] "Optical configuration and analysis of the AMBER/VLTI instrument," A $\& A$ 464, 13.

Shentu, G.-L., Pelc, J. S., Wang, X.-D. et al. [2013] "Ultralow noise up-conversion detector and spectrometer for the telecom band," Opt. Exp. 21, 13986.

Simohamed, L. M., Delage, L. \& Reynaud, F. [1996] "An optical fibre delay line with a $318 \mathrm{~mm}$ stroke," Pure Appl. Opt.: J. Eur. Opt. Soc. A 5, 1005.

Woillez, J., Perrin, G., Lai, O., Coudé du Foresto, V. \& Léna, P. [2001] "OHANA: An optical hawaiian array for nanoradian astronomy," in Proc. 36th Liège Int. Astrophysics Colloquium, p. 139. 